\title{
Tissue microstructural changes in dementia with Lewy bodies revealed by quantitative MRI
}

Li Su ${ }^{a}$, Andrew M. Blamire ${ }^{b}$, Rosie Watson ${ }^{c}$, Jiabao He ${ }^{d}, B^{2}$ enjamin Aribisala ${ }^{e}$, and John T. O’Brien ${ }^{\text {a }}$

a. Department of Psychiatry, University of Cambridge, Addenbrooke's Hospital, Cambridge, UK;

b. Institute of Cellular Medicine \& Newcastle Magnetic Resonance Centre, Newcastle University, Newcastle upon Tyne, UK;

c. Aged Care Department, Royal Melbourne Hospital, Parkville, Australia;

d. The Institute of Medical Sciences, University of Aberdeen, Aberdeen, UK;

e. Division of Clinical Neurosciences, Western General Hospital, University of Edinburgh, Edinburgh, UK

Correspondence to

Dr. Li Su

Department of Psychiatry,

School of Clinical Medicine, University of Cambridge

Box 189, Level E4, Cambridge Biomedical Campus

Cambridge, UK, CB2 OSP

Email: Is514@cam.ac.uk 


\section{Abstract}

Objective: We aimed to characterize dementia with Lewy bodies (DLB) by the quantitative MRI parameters of longitudinal relaxation time (qT1) and transverse relaxation time (qT2). These parameters reflect potential pathological changes in tissue microstructures, which may be detectable noninvasively in brain areas without evident atrophy, so may have potential value in revealing the early neuropathological changes in DLB.

Methods: We conducted a cross-sectional study of subjects with DLB $(\mathrm{N}=35)$ and similarly aged control participants $(\mathrm{N}=35)$. All subjects underwent a detailed clinical and neuropsychological assessment and structural and quantitative 3T MRI. Quantitative MRI maps were obtained using relaxation time mapping methods. Statistical analysis was performed on gray matter qT1 and $\mathrm{qT} 2$ values.

Results: We found significant alterations of quantitative parameters in DLB compared to controls. In particular, qT1 decreases in bilateral temporal lobes, right parietal lobes, basal ganglia including left putamen, left caudate nucleus and left amygadala, and left hippocampus / parahippocampus; qT2 decreases in left putamen and increases in left precuneus. These regions showed only partial overlap with areas where grey matter loss was found, making atrophy an unlikely explanation for our results.

Conclusions: Our findings support that DLB is predominantly associated with changes in posterior regions, such as visual association areas, and 
subcortical structures, and that qT1 and qT2 measurement can detect subtle changes not seen on structural volumetric imaging. Hence, quantitative MRI may compliment other imaging techniques in detecting early changes in DLB and in understanding neurobiological changes associated with the disorder.

(248 words)

\section{Keywords}

dementia, Lewy body disease, quantitative MRI, neuroimaging, VBQ 


\section{Introduction}

Dementia with Lewy bodies (DLB) is the second commonest subtype of degenerative dementia in older people after Alzheimer disease (AD) [1]. It shares common clinical and neuropsychological features with other subtypes of dementia such as AD and Parkinson's disease dementia (PDD), but has a distinctive pattern of pathological changes $[2,3]$. In comparison to AD, DLB has been shown to have less severe atrophy than $A D$, but with a more diffuse and less focal pattern $[4,5]$, with relatively preserved medial temporal lobes $[2,6,7,8]$. One of the most notable characteristics of DLB is visuo-spatial deficit, and imaging studies using mostly PET/SPECT in DLB have highlighted the involvement of occipital $[9,10,11]$, temporoparietal $[1,3,12]$ and subcortical areas $[13,14,15]$. These characteristics of functional rather than structural changes make conventional volumetric MR technique insufficient to fully describe DLB.

There is evidence that other MR parameters, for example, longitudinal relaxation time (qT1) and transverse relaxation time (qT2), may be more sensitive to changes in tissue microstructure, biochemical environment and integrity than macrostructural volume reductions [16]. For example, significant variations in these parameters were found during aging [17] and underlying physiological processes at the cellular and molecular level demonstrated by postmortem histological investigations in transgenic 5xFAD mouse animal model of $A D$ [18]. We therefore hypothesize that quantitative qT1 and qT2 mapping is able to differentiate DLB from healthy controls and indirectly reveal neurobiological abnormalities of DLB at subvoxel scale. 
In this study, we evaluated maps of quantitative modulation of qT1 and qT2 in grey matter, and compared MRI parameters between the DLB and the similarly aged control groups using the voxel-based quantification (VBQ) approach [17]. The main aims of this study were to characterize the neuropathology in DLB based on high-resolution quantitative MR parameter images, and explore their clinical relevance.

\section{Material and methods}

\section{Subject recruitment and assessment}

Thirty-five individuals with probable DLB were recruited from a communitydwelling population of patients referred to local old age psychiatry, geriatric medicine, or neurology services as previously described [2]. Thirty-five controls were recruited from among spouses and friends of the subjects with dementia or from advertisements in local community newsletters. All participants underwent clinical and neuropsychological evaluations with diagnostic procedures previously employed by our group [3]. The research was approved by the local ethics committee. All participants (or, where appropriate, their nearest relative) provided written informed consent.

Assessment of global cognitive measures in all participants involved use of the Cambridge Cognitive Examination (CAMCOG), which incorporates the Mini-Mental State Examination (MMSE) [19]. Motor Parkinsonism was assessed with the Unified Parkinson's Disease Rating Scale Part III (UPDRSIII) [20]. For patients with dementia, neuropsychiatric features were assessed 
with the Neuropsychiatric Inventory (NPI) [21], and function was assessed with the Bristol Activities of Daily Living (Bristol ADL) [22].

\section{MRI data acquisition}

Participants underwent MRI scanning on a 3T Philips Achieva MRI system with an 8-channel receiver head coil. Structural images were acquired using a T1 weighted volumetric sequence (3D MPRAGE, sagittal acquisition aligned with the AC-PC line, $1 \mathrm{~mm}$ isotropic resolution, matrix $240 \times 240 \times 180$, $\mathrm{TR}=9.6 \mathrm{~ms}, \mathrm{TE}=4.6 \mathrm{~ms}$, flip angle $=8^{\circ}$, SENSE factor 2). Quantitative images were acquired using a fast quantitative T1 measurement based on a custom inversion recovery prepared EPI sequence [23] (axial slices, $2 \mathrm{~mm}$ isotropic resolution, matrix $128 \times 128 \times 72, T R=15 \mathrm{~s}, \mathrm{TE}=24 \mathrm{~ms}$, inversion time $\mathrm{TIR}=0.25$ to $2.5 \mathrm{~s}$ in 12 uniform steps); and a fast quantitative multi-echo T2 measurement based on a Gradient and Spin Echo Imaging sequence (2mm isotropic resolution, matrix $128 \times 128 \times 72, T R=4.7 \mathrm{~s}, 8$ spin echoes at $20 \mathrm{~ms}$ spacing, EPI factor 5). Please see Supplementary Information E1 for a validation of quantitative imaging methodology. In addition, a B0 field-map using a dual echo 3D GRE ( $2 \mathrm{~mm}$ isotropic resolution, matrix $128 \times 128 \times 72$, $\mathrm{TR}=27 \mathrm{~ms}, \mathrm{TE}=2.6 / 6.1 \mathrm{~ms}$ ) was acquired.

Statistical Tests of Demographic, clinical, and cognitive measures. Group characteristics were evaluated with Statistical Toolbox of Matlab (www.mathworks.co.uk/products/statistics). Differences in demographic and clinical data were assessed with use of either t-tests for continuous variables or $x^{2}$ tests for categorical measures. For each test statistic, a probability value 
of $p<0.05$ was regarded as significant.

\section{Voxel-based quantification}

MRI data processing was performed in a combined FSL

(fsl.fmrib.ox.ac.uk/fsl/fslwiki) and SPM (www.fil.ion.ucl.ac.uk/spm) based on the previously validated voxel-based quantification (VBQ) procedure [17]. Grey matter volume changes but not other data on this cohort has been previously published [2] and we undertook a similar analysis here to determine whether qT1 and qT2 changes were associated with grey matter atrophy (see Supplementary Information E2). Volumetric structural T1 weighted images were firstly segmented using Gaussian mixture model implemented in the VBM toolbox [24], and brain tissues were classified into grey matter (GM), white matter (WM) and Cerebrospinal fluid (CSF). GM probability maps were non-linearly normalized to standard MNI space (www.mni.mcgill.ca) using the diffeomorphic registration algorithm (DARTEL) [24] in SPM. Raw qT1 and qT2 imaging datasets were corrected for field inhomogeneities using B0 maps and the PRELUDE/FUGUE algorithm in FSL [25]. Then, the bias corrected qT1 and qT2 maps were calculated on voxelby-voxel basis using non-linear least squares fitting to standard single exponential models [16] before further analysis.

In order to minimize the influence of partial volume effect, we used a multimodal approach during the preprocessing stages [17]. The qT1 and qT2 maps were firstly co-registered with the GM probability maps derived from structural T1 images, and then masked using the thresholded GM probability 
maps (at $p>0.5$ ) in each participant's native space. We then transformed the GM maps of qT1 and qT2 MRI parameters into standard MNI space using the participant-specific diffeomorphic parameters estimated from the previous DARTEL procedure. However, we did not apply modulation to these quantitative MRI parameters in order to avoid confound of age and disease related GM volume changes. Finally, all normalized quantitative qT1 and qT2 maps were smoothed with an isotropic Gaussian kernel of $6 \mathrm{~mm}$ full width at half maximum. For the qT1 analysis only, images of three participants in the DLB group were excluded due to the excessive amount of artifact and distortions.

For statistical analysis investigating disease induced regional microstructural alterations between the DLB and control groups, we used the General Linear Model (GLM) with age and gender as covariates. Then, two-tailed t-tests were preformed at each voxel to detect regional difference between the DLB and control groups. The false positive rate was controlled using family-wise error (FWE) correction for multiple comparisons, and thresholded at $p<0.05$ at the cluster level.

\section{Post-hoc region-of-interest analysis}

To quantify the magnitude of changes in qT1 and qT2, we extracted the averaged quantitative MRI values from significant clusters and from two regions, which did not show significant changes (bilateral middle occipital lobes and bilateral middle prefrontal lobes defined using AAL atlas [26]). Here, we used the unsmoothed maps in order to preserve the original values of the 
MR parameters. These plots are not for statistical inference but illustrating the underlying qT1 and qT2 values.

To explore the clinical relevance of qT1 and qT2, in a post-hoc region-ofinterest $(\mathrm{ROI})$ analysis, we have correlated the averaged quantitative MRI values extracted from the significant clusters obtained from the previous group comparison with cognitive and clinical measures. These measurements included the CAMCOG total score (CAM total), also divided into executive function, remote memory (which reflects semantic knowledge), and recent memory (which reflects episodic information) sub scores [19], UPDRS, MMSE, Bristol ADL, NPI and Geriatric Depression Scale (GDS). Multiple comparisons were controlled using Bonferroni correction for the number of cognitive and clinical measures.

\section{Results}

Demographic clinical and cognitive measures

As shown in Table 1, there were no significant differences between DLB and control groups for age, sex and educational level. As expected, on all cognitive and clinical measures (shown in Table 1) DLB patients scored less well that the controls.

\section{$\underline{\text { Insert Table } 1 \text { here }}$}

Group difference in longitudinal relaxation time (qT1)

As shown in Figure $1 \mathrm{~A}$ and Table 2A, we found a significant decrease 
$(p<0.0001, F W E)$ in qT1 for the DLB group compared to controls in bilateral superior and middle temporal lobes, and right angular gyrus. In addition, there was a significant decrease of qT1 in several key subcortical / striatal nuclei such as the left amygadala (extending to hippocampus and parahippocampus) ( $p=0.001, \mathrm{FWE})$, left putamen $(p<0.0001, \mathrm{FWE})$ and left caudate $(p=0.004, \mathrm{FWE})$. There was also a marginally significant reduction in qT1 in the right hippocampus, right putamen and right caudate (not shown). The actual averaged qT1 values from the significant clusters are shown as bar graphs in Figure 1 illustrating the group differences although qT1 in the two control regions in frontal and occipital lobes were not different between groups; see Figure 1B.

$\underline{\text { Insert Table } 2 \text { here }}$

\author{
$\underline{\text { Insert Figure } 1 \text { here }}$
}

Group difference in transverse relaxation time (qT2)

For the transverse relaxation time (qT2), the DLB group showed a significant reduction in left putamen ( $p=0.002, \mathrm{FWE})$; see Figure $2 \mathrm{~A}$ and Table 2B. Moreover, there was also a marginally significant reduction in qT2 in the right putamen (not shown). We also found a significant increase in qT2 for the DLB subjects compared with controls in the left precuneus ( $p=0.021, \mathrm{FWE}$ ); see Figure 2B and Table 2C. Similar to qT1, qT2 in both clusters except for the two control regions were different between groups (see bar graphs in Figure 2). 


\section{$\underline{\text { Insert Figure } 2 \text { here }}$}

Correlation between cognitive / clinical measures and quantitative MRI

A significant correlation was found between the CAMCOG executive function score and the qT1 values in left middle temporal lobe $(r=0.39, p=0.027)$ and left putamen ( $r=0.36, p=0.044)$. In addition, a significant correlation was also found between CAMCOG recent memory score and the left hippocampus extending to amygdala and parahippocapus ( $r=0.53, p=0.0018)$. Finally, a significant inverse correlation was found between Geriatric Depression Scale and the left middle temporal $(r=-0.45, p=0.011)$ and left putamen $(r=-0.42$, $p=0.016)$. No other significant correlation between cognitive / clinical measures and quantitative MRI values was found.

Among the above results, only the correlation between recent memory (but not the remote memory) performance and qT1 in left hippocampus survives the correction for the multiple comparisons (see Figure 3). This relationship was still significant after controlling for age, gender and illness duration as covariates. In addition, DLB patients scored significantly lower than controls in recent $(t(68)=-6.45, p<0.00000001)$ and to a lesser degree but still significantly in remote $(t(68)=-4.27, p<0.00001)$ COMCOG memory tests.

\section{$\underline{\text { Insert Figure } 3 \text { here }}$}

\section{Discussion}

The main findings of this study were tissue water environment changes in 
posterior areas of the cortex and subcortical regions of the brain for DLB. This is consistent with previous MRI [2], SPECT [13,27], PET [28,29,30] and DTI $[3,31]$ studies implicating these regions as most affected by the disease. In particular, we have found alterations in qT1 and qT2 measures in posterior parts of the temporoparietal lobes, and in several subcortical regions such as amygadala, putamen and caudate nucleus. And most strikingly, the majority of these regions do not overlap with where grey matter atrophy was found in DLB (see Supplementary Information E3 and E4). This not only makes atrophy an unlikely explanation for our findings, but suggests that quantitative MRI mapping may compliment other imaging techniques in detecting early changes in DLB when none are demonstrable on conventional structural imaging.

The significant decrease in $\mathrm{T} 1$ relaxation time in the bilateral superior/middle temporal lobes and right parietal regions, e.g. angular gyrus, was associated with a GM volume reduction. This may be related to the memory and attention impairments in DLB. For example, the qT1 value in left hippocampus region (composing of amygdala and parahippocampus) was significantly positively correlated with episodic memory impairment (i.e. CAMCOG recent memory score) but not semantic memory impairment (i.e. CAMCOG remote memory score) in DLB. The absence of a significant correlation between semantic memory and abnormality in temporal cortex is perhaps due to the weaker remote memory deficit in DLB and the relatively more distributed representations of semantic knowledge in the brain. The decrease in qT1 at left middle temporal and putamen was negatively correlated with the severity 
of depression symptoms (at uncorrected level). This implies that the reduction of qT1 in these brain areas may have specific clinical implications. We have also found quantitative MRI parameter (qT2) differences between DLB and controls in precuneus, an area that plays important role in visuospatial processing. Such abnormality in visual and visual association areas may also underpin the clinical and cognitive impairments in patients with DLB, such as recurrent visual hallucinations and visuo-perceptual dysfunction. Consistent with our finding, decreased qT1 is also associated with the layer specific increase in $\beta$-amyloid deposition in 5xFAD mice [18].

Despite the significant clinical manifestations of parkinsonism for DLB, most previous morphometric studies of DLB using structural MRI did not find consistent volume reduction in anatomical areas related to the dopaminergic system. On the one hand, FP-CIT SPECT studies using specific imaging ligands have reported a substantial $40-50 \%$ loss in dopaminergic activity in the striatum [27]. On the other hand, Cousins et al [15] have only reported an approximately $13 \%$ volume reduction in bilateral putamen in DLB compared to controls. With the minimal reduction in subcortical volume, previous MRI studies on GM volume changes did not fully reflect the notable dopamine transporter deficiencies found in DLB.

However, we have identified several subcortical regions in the brain that are potentially associated with dopamine functions. In comparison to control subjects, the averaged qT1 and qT2 values in putamen were decreased by $45 \%$ and $52 \%$ respectively with DLB suggesting that these local tissue 
alterations may be due to Lewy body related pathology and agreeing with findings from FP-CIT SPECT studies [27]. In addition, we have shown that qT1 value in left putamen was correlated with executive function deficit in DLB (at the uncorrected level). During healthy aging, qT2* (a quantitative MRI parameter related to qT2) has also been found to be negatively correlated with age in a wide range of brain areas, in particular in bilateral putamen, reflecting an increased level of iron [32]. In parkinson's disease, lower qT2* in putamen is also associated with more severe motor symptoms [33]. In addition, we have shown that qT1 parameters were decreased in the left caudate nucleus by $68 \%$ with DLB. This finding is consistent with the volume reduction in this area and with the known degeneration of the nigrostriatal dopamine pathway associated with DLB.

In terms of determining the possible underlying biophysical interpretation of these quantitative MRI parameters, qT1 is predominantly influenced by water content but also relates to the degree of myelination $[34,35,36]$. Both qT1 and qT2 are also sensitive to the level of iron (or other paramagnetic ions in the tissue) $[37,38]$. It is difficult to say precisely what causes qT1 or qT2 to change since they are affected by a range of processes, e.g. demyelination, inflammation, iron, ischemia, edema, and so on. It remains to be seen whether changes in quantitative MRI values reflect abnormalities in the local tissue environment associated with neurodegenerative diseases in general, or whether they may indicate a more specific degenerative process. Nonetheless, the potential ability of quantitative MRI in detecting early changes of tissue property caused by neurodegenerative disease without 
evident atrophy is clinically promising. Compared to nuclear imaging and structural MRI, quantitative $\mathrm{MRI}$ is a new and versatile diagnostic tool in investigating neurobiological changes in DLB, with potential for the early detection of DLB since it is not only a noninvasive method, but can also provide more subtle information on local pathology than morphometric measures.

A potential weakness of this approach is the relatively low $2 \mathrm{~mm}$ isotropic resolution compared to the cortical thickness. Other studies have achieved finer resolution in healthy controls but at the expense of substantially longer times, which are challenging for this patient group [36]. Our scans were acquired with scan times of less than 4 minutes each making them acceptable in ageing populations. At $2 \mathrm{~mm}$ resolution there is a potential for partial volume effects, particularly with CSF, which can substantially skew quantitative values [39]. Such partial volume effects would cause significant increases in qT1 and qT2 and would be most evident in regions of cortical atrophy. As our data show reduced values and do not coalign with the previously described pattern of atrophy in this patient cohort [2] we are confident that these changes reveal true disease related, parametric changes in the underlying gray matter (see Supplementary Information E5).

\section{Conclusions}

In conclusion, we have shown that quantitative MRI is applicable in both understanding the neurobiology of the disease and has the potential in clinical diagnosis. Further research should investigate at what stages in the disease 
qT1 and qT2 changes start to occur, and how they alter with disease progression.

\section{Acknowledgment}

We thank Nikolaus Weiskopf for insightful discussion on voxel-based quantification methods. The study was funded by the Sir Jules Thorn Charitable Trust (grant ref: 05/JTA) and was supported by the National Institute for Health Research (NIHR) Newcastle Biomedical Research Centre and the Biomedical Research Unit in Lewy Body Dementia based at Newcastle upon Tyne Hospitals National Health Service (NHS) Foundation Trust and Newcastle University and the NIHR Biomedical Research Centre and Biomedical Research Unit in Dementia based at Cambridge University Hospitals NHS Foundation Trust and the University of Cambridge. The views expressed are those of the authors and not necessarily those of the NHS, the NIHR or the Department of Health.

\section{Author contributions}

L.S. designed the study, carried out the statistical analysis and wrote the manuscript. A.M.B. codesigned the study, assisted with data interpretation, assisted with writing of the manuscript, and obtained funding for the project. R.W. codesigned the study, recruited and assessed study participants and reviewed the manuscript. J.H. and B.A. were involved with design of the imaging protocol and reviewed the manuscript. J.T.O. codesigned the study, provided subject diagnostic rating, assisted with the development of the research question and with writing of the manuscript, and obtained funding for 
the project.

\section{Disclosure}

L. Su, A. Blamire, R. Watson, J. He and B. Aribisala report no disclosures. J. O'Brien has been a consultant for GE Healthcare, Servier, and Bayer Healthcare and has received honoraria for talks from Pfizer, GE Healthcare, Eisai, Shire, Lundbeck, Lilly, and Novartis. 


\section{References}

1. McKeith IG, Dickson DW, Lowe J, et al. Diagnosis and management of dementia with Lewy bodies: third report of the DLB Consortium. Neurology $2005 ; 65: 1863-1872$.

2. Watson R, O'Brien JT, Barber R, Blamire AM. Patterns of gray matter atrophy in dementia with Lewy bodies: a voxel-based morphometry study. International Psychogeriatrics 2012; 24: 532-543.

3. Watson R, Blamire AM, Colloby SJ, et al. Characterizing dementia with Lewy bodies by means of diffusion tensor imaging. Neurology 2012; 79:906914.

4. Burton, EJ et al. Patterns of cerebral atrophy in dementia with Lewy bodies using voxel-based morphometry. Neurolmage 2002; 17:618-630.

5. Beyer MK, Larsen JP and Aarsland D. Gray matter atrophy in Parkinson disease with dementia and dementia with Lewy bodies. Neurology 2007; 69:747-754.

6. Whitwell JL et al. Focal atrophy in dementia with Lewy bodies on MRI: a distinct pattern from Alzheimer's disease. Brain 2007; 130:708-719.

7. Lippa CF, Johnson R and Smith TW. The medial temporal lobe in dementia with Lewy bodies: a comparative study with Alzheimer's disease. Annals of 
Neurology 1998: 43:102-106.

8. Burton EJ et al. Medial temporal lobe atrophy on MRI differentiates Alzheimer's disease from dementia with Lewy bodies and vascular cognitive impairment: a prospective study with pathological verification of diagnosis. Brain 2009; 132:195-203.

9. Lobotesis K, Fenwick JD, Phipps A, et al. Occipital hypoperfusion on SPECT in dementia with Lewy bodies but not AD. Neurology 2001; 56:643649.

10. Minoshima S, Foster NL, Petrie EC, et al. Neuroimaging in dementia with Lewy bodies: metabolism, neurochemistry, and morphology. J Geriatr Psychiatry Neurol 2002; 15:200-209.

11. Hanyu H, Shimizu S, Hirao K, et al. Differentiation of dementia with Lewy bodies from Alzheimer's disease using Mini-Mental State Examination and brain perfusion SPECT. J Neurol Sci 2006; 250:97-102.

12. Sanchez-Castaneda C, Rene R, Ramirez-Ruiz B, et al. Frontal and associative visual areas related to visual hallucinations in dementia with Lewy bodies and Parkinson's disease with dementia. Movement Disorders 2010; 25:615-622.

13. Piggott MA, Marshall EF, Thomas N, et al. Striatal dopaminergic markers 
in dementia with Lewy bodies, Alzheimer's and Parkinson's diseases: rostrocaudal distribution. Brain 1999; 122 (Pt 8):1449-1468.

14. McKeith I, O'Brien J, Walker Z, et al. Sensitivity and specificity of dopamine transporter imaging with 123I-FP-CIT SPECT in dementia with Lewy bodies: a phase III, multicentre study. Lancet Neurol 2007; 6:305-313.

15. Cousins DA, Burton EJ, Burn D et al. Atrophy of the putamen in dementia with Lewy bodies but not Alzheimer's disease: an MRI study. Neurology 2003; 61:1191-1195.

16. Tofts P. Quantitative MRI of the brain: measuring changes caused by disease. John Wiley \& Sons Ltd 2003.

17. Draganski B, Ashburner J, Hutton C, et al. Regional specificity of MRI contrast parameter changes in normal ageing revealed by voxel-based quantification (VBQ). Neurolmage 2011; 55:1423-1434.

18. Spencer NG, Bridgesb LR, Elderfield K, et al. Quantitative evaluation of MRI and histological characteristics of the 5xFAD Alzheimer mouse brain. Neurolmage 2013; 76:108-115.

19. Folstein M, Folstein S, McHugh P. "Mini-mental state": a practical method for grading the cognitive state of patients for the clinician. J Psychiatr Res $1975 ; 12: 189-198$. 
20. Fahn S, Elton R, members of the UPDRS Development Committee. Unified Parkinson's Disease Rating Scale. In: Fahn S, Marsden C, Calne D, Goldstein M, eds. Recent Developments in Parkinson's Disease. Florham Park: Macmillan Health Care Information; 1987:153-164.

21. Cummings JL, Mega M, Gray K, et al. The Neuropsychiatric Inventory: comprehensive assessment of psychopathology in dementia. Neurology $1994 ; 44: 2308-2314$

22. Bucks RS, Ashworth DL, Wilcock GK, Siegfried K. Assessment of activities of daily living in dementia: development of the Bristol Activities of Daily Living Scale. Age Ageing 1996; 25:113-120.

23. Clare $\mathrm{S}$, Jezzard P. Rapid T-1 mapping using multislice echo planar imaging. Magnetic Resonance in Medicine 2001; 45:630-634.

24. Ashburner J. A fast diffeomorphic image registration algorithm. Neurolmage 2007; 38:95-113.

25. Jenkinson M, Beckmann CF, Behrens TEJ, et al. FSL. Neurolmage 2012; 62(2):782-790.

26. Tzourio-Mazoyer N, Landeau B, Papathanassiou D, et al. Automated Anatomical Labeling of Activations in SPM Using a Macroscopic Anatomical 
Parcellation of the MNI MRI Single-Subject Brain. Neurolmage 2002; 15:273289.

27. O'Brien JT, Colloby S, Fenwick J, et al. Dopamine transporter loss visualized with FP-CIT SPECT in the differential diagnosis of dementia with Lewy bodies. Arch Neurol 2004; 61:919-925.

28. Klein JC, Eggers C, Kalbe E, et al. Neurotransmitter changes in dementia with Lewy bodies and Parkinson disease dementia in vivo. Neurology 2010; $74: 885-892$

29. Edison P, Rowe CC, Rinne JO, et al. Amyloid load in Parkinson's disease dementia and Lewy body dementia measured with [11C]PIB positron emission tomography. J Neurol Neurosurg Psychiatry 2008; 79:1331-1338.

30. Shimada H, Hirano S, Shinotoh $\mathrm{H}$, et al. Mapping of brain acetylcholinesterase alterations in Lewy body disease by PET. Neurology 2009; 73:273-278.

31. Kantarci K, Avula R, Senjem ML, et al: Dementia with Lewy bodies and Alzheimer disease: neurodegenerative patterns characterized by DTI. Neurology 2010; 74:1814-1821.

32. Callaghan MF, Freund P, Anderson E, at al. Widespread age-related differences in the human brain microstructure revealed by quantitative 
magnetic resonance imaging. Neurobiology of Aging

2014;doi:10.1016/j.neurobiolaging.2014.02.008.

33. Bunzeck N, Eckart C, Singh-Curry V, et al. Motor phenotype and magnetic resonance measures of basal ganglia iron levels in Parkinson's disease. Parkinsonism and Related Disorders 2013; 19(12):1136-1142.

34. Gelman N, Ewing JR, Gorell JM et al. Interregional variation of longitudinal relaxation rates in human brain at $3.0 \mathrm{~T}$ : relation to estimated iron and water contents. Magn. Reson. Med. 2001; 45:71-79.

35. MacKay A, Whittall K, Adler $\mathrm{J}$ et al. In vivo visualization of myelin water in brain by magnetic resonance. Magn. Reson. Med. 1994; 31:673-677.

36. Lutti A, Dick F, Sereno Ml, et al., Using high-resolution quantitative mapping of R1 as an index of cortical myelination. Neuroimage 2014; 93:176188.

37. Fukunaga M, Li, TQ, van Gelderen $P$ et al. Layer-specific variation of iron content in cerebral cortex as a source of MRI contrast. Proc. Natl Acad. Sci. USA 2010; 107:3834-3839.

38. Gelman N, Ewing JR, Gorell JM et al. Interregional variation of longitudinal relaxation rates in human brain at $3.0 \mathrm{~T}$ : relation to estimated iron and water contents. Magn. Reson. Med. 2001; 45:71-79. 
39. Aribisala BS, He J, Blamire AM. Comparative study of standard space and real space analysis of quantitative MR brain data. J Magn Reson Imaging 2011; 33(6):1503-1509. 